\title{
Attentional focus, perceived target size, and movement kinematics under performance pressure
}

\author{
Rob Gray ${ }^{1} \cdot$ Rouwen Cañal-Bruland ${ }^{2}$
}

Published online: 2 May 2015

(C) Psychonomic Society, Inc. 2015

\begin{abstract}
The present study examined the relationship between attentional focus, perceived hole size, and radial putting error in a golf task. Twenty-five experienced golfers were asked to produce size estimates immediately after completing a putt. To assess their attentional focus, one of two secondary tasks (chosen randomly) was performed next. In the Hole task, participants were asked to indicate whether a sound played during their putting stroke was presented to the left or right of the hole. In the Club task, they were asked to indicate whether the sound occurred closer to the beginning or end of their backswing. Participants completed three phases: a no pressure pretest, a pressure phase, and a no pressure posttest. There were substantial individual differences in the effects of pressure on putting kinematics: 11 golfers (designated the Choke group) showed significant changes in kinematic variables and heart rate, and 14 golfers (designated the Clutch group) showed no significant change in these variables. For the Choke group, putting error and the accuracy on the Club task significantly increased during the pressure phase while size estimates and accuracy on the Hole task significantly decreased. There were no significant changes in any of these variables for the Clutch group. These findings provide further evidence for the attentional accentuation hypothesis of action-specific effects.
\end{abstract}

Rob Gray

robgray@asu.edu

Rouwen Cañal-Bruland

r.canalbruland@vu.nl

1 Human Systems Engineering, Arizona State University Polythechnic, Santa Catalina Hall, 7271 E. Sonoran Arroyo Mall, Mesa, AZ, USA

2 VU University Amsterdam, MOVE Research Institute Amsterdam, Faculty of Human Movement Sciences, Van der Boechorststraat 9 , 1081 BT Amsterdam, The Netherlands
Keywords Action $\cdot$ Perception $\cdot$ Performance $\cdot$ Sports

Recent research has demonstrated repeatedly that performance of a skill that involves acting on a goal object (e.g., a ball to be hit) can influence ones perceptual judgments of that object. For example, Witt and Proffitt (2005) reported that softball players with a higher batting average in a recently completed set of games judge the ball to be larger than do players with a lower average. Similar effects also have been reported for perceptual judgments of hole size in golf (Cañal-Bruland et al. 2011; Witt et al. 2008), upright size in field goal kicking in football (Witt and Dorsch 2009), target size in dart throwing (Cañal-Bruland et al. 2010; Wesp et al. 2004), target size in archery (Lee et al. 2012), and estimates of runway size in aviation (Gray et al. 2014). These "action-specific effects" are all examples of an individual's ability to interact with goal objects in the environment (as indexed by their performance success) changing the way in which these objects are perceptually judged. These findings are consistent with the theoretical viewpoint that perception directly specifies the relationship between the perceiver and the environment rather than solely being based on the physical properties of objects (Gibson 1950; Proffitt 2006).

Despite the growing body of research demonstrating action-specific effects on perception, the underlying mechanisms have not been clearly identified. Alternative accounts to the embodied perception explanation described above have been put forward including ones based on experimental demands (Durgin et al. 2009) and memory effects (Cooper et al. 2012). In the present study, we focus on an additional alternative explanation for action-specific effects: namely attentional accentuation. Cañal-Bruland and van der Kamp (2009) have proposed that these action-specific effects are consistent with the perceptual accentuation hypothesis (Bruner 1957), which claims that when a person intends to act on object and directs 
their attention to it, the task-relevant object becomes accentuated so that it stands out from other task-irrelevant objects (Balcetis and Dunning 2010). Evidence to support this hypothesis comes from a recent study by Cañal-Bruland et al. (2011) in which it was demonstrated that action-specific changes in the perceived size of a golf hole do not occur when a golfer is prevented from focusing their attention on the hole (because it was occluded) or when golfers are required to shift attention between an intermediate object and the target object (due to a requirement to hit a ball through a gate on its way to the hole).

Further support for this hypothesis comes from research that has examined action-specific perception under conditions of high anxiety (Cañal-Bruland et al. 2010). In this study, participants threw darts at a target and produced a judgment of target size. Under conditions of low anxiety (throwing while standing on the ground), there was a significant positive correlation between judged size and throwing performance, whereas under conditions of high anxiety (throwing while suspended above the ground), there was no correlation. The authors argued that the lack of effect in the high anxiety condition was due to the fact that the performer's attention was drawn away from the target (and thus accentuation did not occur). A shift in attention of this type is consistent with both of the main theories of what is thought to occur to a performer under conditions of high anxiety/pressure: distraction theory and self-monitoring (reviewed in Beilock and Gray 2007).

\section{Study goals}

The goal of the present study was to further investigate the role of attention in action-specific perception by expanding on the anxiety study conducted by Cañal-Bruland et al. (2010). In this previous study, only perceived size and performance outcomes were measured. Thus, there was no direct evidence that the change in action-specific perception was due to a shift in the performer's attentional focus. However, previous research has demonstrated direct links between performance success, attentional focus, and movement kinematics under pressure conditions. For example, using a baseball batting task with college players, Gray (2004) found that a combination of competitive and evaluation pressure resulted in poorer batting performance, an increase in skillfocused (internal) attention (i.e., batters performed better in a secondary task that involved judging the direction of bat movement), and a significant change in kinematics (specifically, an increased amount of variability in the timing of the different stages of their swing). Similarly, in golf putting an increase in skill-focused attention (produced either by pressure or the introduction of a secondary task that involves making judgments about the movement of the putter) can produce both an increase in putting error and a change in putting kinematics (Beilock and Gray 2012; Gray, Allsop \& Williams, 2013). In this case, the kinematic changes were an increase in club-ball impact velocity, a decrease in the time to peak speed, and a change in the relationship between downswing amplitude and putting distance. It is notable that all of these kinematic characteristics are typical of novice golfers (Delay, et al. 1997). See also Lohse et al. (2010) and Cooke et al. (2010) for similar findings. To our knowledge, no previous studies have examined how these changes in movement kinematics are related to the perceived size of the target object. Furthermore, although links between attentional focus and perceived size have been inferred through looking at fixation patterns (Wood et al. 2013; Gray et al. 2014), to our knowledge no previous studies have directly manipulated attentional focus and measured the effect on perceived size.

In the present study, we sought to investigate the link between perceived size, performance outcomes, attentional focus, and movement kinematics in a golf putting task by measuring each of these variables and assessing how they change under pressure. Specifically, in conjunction with performing a putting task, participants were asked to estimate the size of the golf hole under nonpressure pre- and posttests and a pressure condition. The pressure condition involved a combination of competitive (monetary incentive) and evaluative (performance recorded and results distributed) pressure. The attentional probe methodology was used to assess the extent to which participants were focusing their attention on the hole or the movement of their club-head (Gray 2004; Gray and Allsop 2013). Finally, time to peak speed (TTPS) was used as the primary kinematic measure, because it has been shown to be related to skill level in golf and be influenced by pressure (Beilock and Gray 2012). The experiment was designed to test the following predictions:

1) Putting error would be significantly higher in the pressure phase as compared to the nonpressure phases.

2) Accuracy on the hole-related attentional task would be significantly lower and accuracy on the club-related attentional task significantly higher in the pressure phase reflecting an inward shift of attention (consistent with the effects found by Gray (2004) described above).

3) TTPS would be significantly shorter in the pressure phase as compared to the nonpressure phases.

4) Perceived hole size would be significantly smaller in the pressure phase compared with the nonpressure phases. This effect was predicted, because we expected that attention would be shifted away from the hole in the pressure situation resulting in decreased attentional accentuation.

\section{Methods}

\section{Participants}

A total of $25(17 \mathrm{M}, 8 \mathrm{~F})$ right-handed, experienced golfers enrolled from the School of Sport, Exercise \& Rehabilitation 
Science program at the University of Birmingham participated in the study. Their mean age, mean handicap, and mean number of years competitive playing experience were $20.1(S E=0.4)$ years, $7.3(S E=0.6)$ strokes, and $6.2(S E=0.8)$ years respectively. Ethical approval was granted by the Science, Technology, Mathematics and Engineering (STEM) Ethical Review Committee at The University of Birmingham.

\section{Apparatus}

A McGregor M220 ${ }^{\mathrm{TM}}$, 35-in (88.9 cm), right-handed putter and Wilson Ultra ${ }^{\mathrm{TM}}$ golf balls (size 1.68 in $[4.27 \mathrm{~cm}]$ ) were used. The artificial putting mat had a width of $1.4 \mathrm{~m}$ and a length of $4.6 \mathrm{~m}$. The putting task required participants to putt a golf ball towards a $16.5-\mathrm{cm}$ diameter red circle placed on the surface of the green. Participants were instructed to "stop the ball as close as possible to the centre of the target circle." All putts were made from a distance of $2.5 \mathrm{~m}$. The $\mathrm{x} / \mathrm{y} / \mathrm{z}$ location and angle of the putter head was recorded by mounting a Fastrak (Polhemus ${ }^{\mathrm{TM}}$ ) position tracker sensor weighing $10 \mathrm{~g}$ on the back side of the putter.

Estimates of perceived hole size were obtained using the method used by Wood et al. (2013) by asking participants to draw (using a computer mouse) life-sized replicas of the target circle on a monitor using PowerPoint presentation software. The monitor was positioned perpendicular to the target circle at a distance equal to the putting distance $(2.5 \mathrm{~m})$.

Auditory stimuli for the attentional probes were presented via two speakers (Logitech Model X140) placed at the end of the putting surface at a distance of $3.5 \mathrm{~m}$ from the participant. The speakers were placed $10 \mathrm{~cm}$ on either side of the hole, a distance determined in pilot experiments.

Heart rate served as an index of sympathetic nervous system activity and was measured using a heart rate receiver unit (Polar Electro S625X, Polar CIC Inc., USA), which was connected to a transmitter (Polar Electro coded 31, Polar CIC Inc.) with moistened electrodes positioned across the lowermid thorax. Average heart rate was calculated for the different phases described below.

\section{Procedure}

The experiment was divided into four phases all completed within one 1.5-hour session: practice, pretest, pressure, and posttest. Participants were given 10-minute breaks between each phase. In all test phases, participants saw the final position of the ball on the green.

Practice phase During this phase, participants took 20 putts under normal, single-task conditions. These trials allowed participants to become comfortable with the sensor mounted on the back of the putter as well as to familiarize themselves with our putting task. They were not required to perform the hole size estimate during this phase. Following each putt, the experimenter measured the radial distance between the center of the target and the final position of the ball (in $\mathrm{cm}$ ).

Position tracker data from these practice trials was used to measure the timing of the putting stroke for each participant (Beilock and Gray 2012). This timing information was later used to control stimulus presentation for one of the secondary tasks as described below. For each putting stroke, we determined the instant in time when the putter began moving $\left(\mathrm{START}_{\mathrm{t}}\right)$ and the instant in time when the top of the backstroke was reached $\left(\mathrm{BACK}_{\mathrm{t}}\right)$. We then calculated the mean values of these variables for each participant.

Pretest phase During this phase, the hole size estimation task was first explained to each participant and was practiced five times (without putting). The two secondary tasks were next explained to the participant and practiced five times each (without making the hole size judgment). Both secondary tasks involved the presentation of auditory cues, which were pure tones with a frequency of $500 \mathrm{~Hz}$ and duration of $150 \mathrm{~ms}$. The tones were presented via one of the two speakers placed on either side of the hole. Position tracker data taken from the practice trials was used to present the cue at a random time during the participant's backswing, i.e., interval between $\mathrm{START}_{\mathrm{t}}$ and $\mathrm{BACK}_{\mathrm{t}}$. If the tone was not presented in this interval or occurred at the midpoint of the backswing, the data from that trial was discarded and the trial was re-run. The two secondary tasks were modelled after Beilock and Gray (2012) and were as follows:

Hole task For this task, participants were required to make a two alternative forced choice (2AFC) judgment about whether the tone presented was played from the speaker to the left or to the right or the hole. Responses were made verbally (by saying "left" or "right") after the putt was complete (see below).

Club task For this task, participants were required to make a 2AFC judgment about whether the tone occurred close to the start or the end or their backswing by saying "start" or "end" after the putting stroke was complete. The tone was presented in the backswing, because our previous research has shown that this is the phase of the putting stroke that is most sensitive to attentional manipulations and golfers can perform this task with a relatively high degree of accuracy (Beilock and Gray 2012).

After all of the practice trials were completed, participants completed 30 putts. For each putt, participants first completed the stroke and observed where the ball stopped. After each putt, they were then asked to perform the hole size estimation as described above. Finally, they were given a verbal prompt by the experimenter as to which secondary task response was required. The 30 putts were divided into 12 putts with a Hole Task prompt, 12 putts with a Club Task prompt, and 6 putts 
with a "No Task" prompt for which no secondary task response was required. This ratio of prompt types was similar to that used in our previous study (Beilock and Gray 2012). The order of these prompts was chosen randomly (without replacement) on each trial for each participant. The speaker side on which the tone was presented also was chosen randomly. Participants were not given any feedback about their hole estimation or the secondary task response.

Note that we chose to have participants complete the secondary task after they made the hole size estimate to avoid the possibility that the former would influence the latter. The secondary task prompts were given after the putt was complete and $20 \%$ of trials involved no secondary task judgment with the goal of measuring where the participant's attention was focused rather than directing it to a specific location via the secondary task. Because the participant did not know before or during the putt whether they would be asked to make the hole or club judgment, there was no advantage to shifting their attentional focus to one or the other location (Beilock and Gray 2012; Gray 2004; Gray and Allsop 2013). These two secondary tasks were chosen to allow for direct comparison with our previous research. How they might directly interact with the hole size estimation task is discussed in more detail below.

Pressure phase This phase was identical to the pretest phase except that participants were given instructions prior to putting designed to increase competitive and evaluation pressure. Before beginning putting, participants read the following script:

We're now moving into a competition phase. Your objective in the competition is still to putt the ball as close to the marker as possible. However, throughout the experiment you have so far accumulated 180 points. For every putt that finishes more than $5 \mathrm{~cm}$ from the marker, you will lose 10 points. Prize money of $£ 50, £ 25$, and $£ 10$ is up for grabs, for 1 st, 2 nd, and 3rd place. How many points you manage to hold on to determines your position on the leader board. All the results will be emailed to everyone who takes part in the study and will be displayed on the notice board in the school atrium. So, everyone will know how everyone else performs. No pressure then "said sarcastically"...good luck!

Note, this procedure is identical to that used in our previous study on the effects of pressure on golf performance and has proven to produce significant pressure effects (Gray et al. 2013).

The Immediate Anxiety Measures Scale (IAMS; Thomas et al. 2002) was used to assess participants' intensity of cognitive and somatic anxiety. The questionnaire was composed of two items measuring the extent to which participants felt cognitively anxious (I was cognitively anxious) and somatically anxious (I was somatically anxious). Responses were made on a 7-point Likert-type scale ranging from 1 (not at all) to 7 (extremely). This scale was completed at the end of each phase of the experiment.

Posttest phase The posttest phase was identical to the pretest phase.

\section{Data analysis}

Manipulation check To determine to what extent our pressure manipulation had the desired effect, the cognitive and somatic anxiety ratings for the IAMS and the heart rate data were subjected to separate one-way repeated measures ANOVA with phase (pretest, pressure, posttest) as the independent variable.

Task performance There were four main dependent variables that were analyzed: mean radial error (MRE), mean perceived hole size (MPS), percentage correct for the Hole secondary task (\%Hole), and percentage correct for the Club secondary task (\%Club). Each of these variables was first analyzed using separate one-way repeated measures ANOVA with phase as the independent variable.

Putting kinematics To further evaluate the effects of pressure on putting performance, we also analyzed time to peak speed (TTPS) and velocity at impact IVI, as these kinematic variables were found to change significantly in pressure conditions in our previous study (Gray et al., 2013).

\section{Results}

\section{Data clustering}

A preliminary analysis of the data revealed that - as in our previous study (Gray et al., 2013) — there were large individual differences in the response to pressure. Specifically, some participants showed a significant change in behavior under pressure while others did not. Therefore, we decided to analyze the data separately for these two groups. To divide participants, we used a k-means cluster analysis with five classification variables: change in cognitive anxiety, change in somatic anxiety, change in heart rate, change in TTPS, and change in VI. The first three variables were used, because they are commonly employed measures of anxiety (Beilock and Gray 2007), whereas the two kinematic variables were used because, as described above, previous research has shown that pressure can result in a decrease in TTPS and an increase in the velocity at impact (VI) of the putting stroke (Gray et al., 2013). This analysis resulted in two data clusters: cluster 1 with 11 participants and cluster 2 with 14 participants. The 
significant classification variables were change in TTPS $[F(1$, $23)=172.7, p<0.001]$, change in $\mathrm{VI}[\mathrm{F}(1,23)=15.8, p=$ $0.001]$, and change in $\operatorname{HR}[\mathrm{F}(1,23)=68.9, p \leq 0.001]$. The values for these variables are shown in Table 1 . Both change in cognitive and change in somatic anxiety were not significant (both $p>0.5$ ). The final cluster centers for the significant variables were as follows. Cluster $1:-0.21$ (TTPS), 0.11 (VI), and 8.45 (HR); Cluster 2: -0.01 (TTPS), -0.03 (VI), and 0.79 (HR). Note that center values in cluster 1 (lower TTPS, higher VI, and higher HR) are all consistent with being affected by the performance pressure, whereas the comparatively small changes in these variables for cluster 2 suggest that participants in this group were not affected by pressure.

Therefore, following convention in pressure research (Otten 2009), we refer to cluster 1 as "Choke" and cluster 2 as "Clutch" below.

\section{Manipulation check}

Figure 1a shows the mean heart rates for the different phases of the experiment separated for the Choke and Clutch groups.

Table 1 Significant variables in cluster analysis

\begin{tabular}{|c|c|c|c|c|}
\hline Participant & Cluster & $\begin{array}{l}\text { Change in } \\
\text { TTPS (sec) }\end{array}$ & $\begin{array}{l}\text { Change in } \\
\text { VI }(\mathrm{m} / \mathrm{s})\end{array}$ & $\begin{array}{l}\text { Change in } \\
\text { HR (BPM) }\end{array}$ \\
\hline 1 & 2 & -0.01 & 0.03 & 2.00 \\
\hline 2 & 2 & 0.02 & -0.40 & 0.00 \\
\hline 3 & 2 & -0.01 & 0.01 & -5.00 \\
\hline 4 & 2 & 0.01 & -0.02 & 1.00 \\
\hline 5 & 2 & 0.0 & 0.05 & 3.00 \\
\hline 6 & 2 & 0.01 & 0.03 & -2.00 \\
\hline 7 & 2 & -0.02 & 0.05 & 1.00 \\
\hline 8 & 2 & -0.04 & -0.06 & 4.00 \\
\hline 9 & 2 & 0.00 & -0.03 & -2.00 \\
\hline 10 & 2 & -0.01 & 0.03 & 3.00 \\
\hline 11 & 2 & 0.05 & 0.02 & 4.00 \\
\hline 12 & 2 & 0.02 & -0.04 & 2.00 \\
\hline 13 & 2 & -0.01 & -0.09 & 3.00 \\
\hline 14 & 2 & 0.09 & -0.03 & -3.00 \\
\hline 15 & 1 & -0.15 & 0.11 & 8.00 \\
\hline 16 & 1 & -0.29 & 0.09 & 11.00 \\
\hline 17 & 1 & -0.19 & 0.12 & 8.00 \\
\hline 18 & 1 & -0.24 & 0.14 & 8.00 \\
\hline 19 & 1 & -0.18 & 0.08 & 9.00 \\
\hline 20 & 1 & -0.28 & 0.11 & 10.00 \\
\hline 21 & 1 & -0.21 & 0.15 & 8.00 \\
\hline 22 & 1 & -0.16 & 0.13 & 9.00 \\
\hline 23 & 1 & -0.19 & 0.11 & 6.00 \\
\hline 24 & 1 & -0.24 & 0.07 & 7.00 \\
\hline 25 & 1 & -0.15 & 0.08 & 9.00 \\
\hline
\end{tabular}

These data were analyzed with a 2 × 3 mixed ANOVA with group (Choke, Clutch) and phase (pretest, pressure, posttest) as factors. This analysis revealed significant main effects of group $\left[\mathrm{F}(1,23)=15.4, p=0.01, \eta_{\mathrm{p}}^{2}=0.40\right]$ and phase $\left[\mathrm{F}(2,46)=23.2, p<0.001, \eta_{\mathrm{p}}^{2}=0.50\right]$ and a significant group $\mathrm{x}$ phase interaction $[\mathrm{F}(2,46)=12.9, p<0.001$, $\left.\eta_{\mathrm{p}}^{2}=0.35\right]$. A post-hoc $t$ test revealed that the mean heart rate was significantly higher in the pressure phase for the Choke group compared with the clutch group: $\mathrm{t}(23)=6.15, p<0.001$.

Figure $1 \mathrm{~b}$ shows the mean cognitive and somatic anxiety ratings for the different phases of the experiment separated for the Choke and Clutch groups. These data were analyzed with separate 2 × 3 mixed ANOVAs with group (Choke, Clutch) and phase (pretest, pressure, posttest) as factors. This analysis revealed significant effects of phase for both
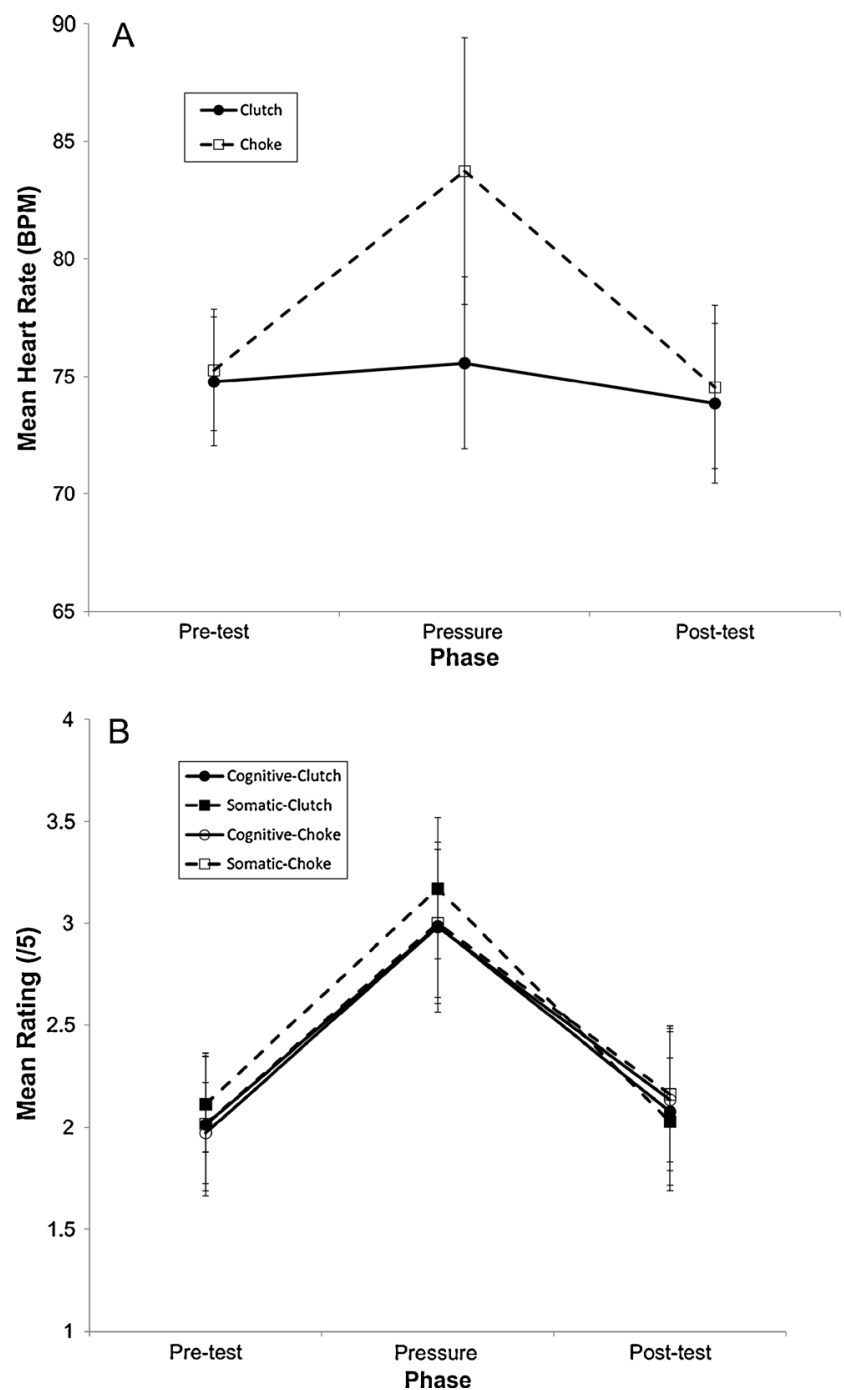

Fig. 1 a Mean heart rates for the different phase of the experiment and different groups. b Mean cognitive and somatic anxiety ratings for the different phases of the experiment and the different groups. Error bars show $\pm 1 \mathrm{SE}$ 
cognitive $\left[\mathrm{F}(2,46)=48.5, p<0.001, \eta_{\mathrm{p}}^{2}=0.68\right]$ and somatic $\left[\mathrm{F}(2,48)=74.8, p<0.001, \eta_{\mathrm{p}}^{2}=0.76\right]$ anxiety. For both anxiety ratings, neither the main effect of group nor the group $\mathrm{x}$ phase interaction were significant (all $p>0.2, \eta_{\mathrm{p}}^{2}<1$ ). In other words, both physiological (i.e., heart rate) and kinematic measures differed between the two groups as predicted, whereas the self-reported cognitive and somatic anxiety measures did not differ.

\section{Putting performance}

Figure 2 shows the MRE for the two groups across the different phases of the experiment. These data were analyzed using a $2 \times 3$ mixed ANOVA with group (Choke, Clutch) and phase as factors. This analysis revealed significant main effect of phase $\left[\mathrm{F}(2,46)=22.4, p<0.001, \eta_{\mathrm{p}}^{2}=0.49\right]$ and a significant group x phase interaction $\left[\mathrm{F}(2,46)=3.4, p=0.04, \eta_{\mathrm{p}}^{2}=0.13\right]$. Post-hoc $t$ tests revealed that MRE was significantly higher in the pressure phase for Choke group: $\mathrm{t}(23)=2.79, p=0.01$. All other comparisons were not significant.

\section{Hole size estimates}

Figure 3 shows the MPS for the two groups across the different phases of the experiment. The $2 \times 3$ ANOVA performed on these data revealed a significant main effect of phase $\left[\mathrm{F}(2,46)=6.1, p=0.004, \eta_{\mathrm{p}}^{2}=0.21\right]$ and a significant group $\mathrm{x}$ phase interaction $\left[\mathrm{F}(2,46)=3.7, p=0.03, \eta_{\mathrm{p}}^{2}=0.14\right]$. Post-hoc $t$ tests revealed that for the Choke group MPS was significantly smaller in pressure phase compared with both the pretest $[\mathrm{t}(10)=-3.55, p=0.005]$ and the posttest $[\mathrm{t}(10)=-3.73, p=0.004]$ phases. All other comparisons were not significant.

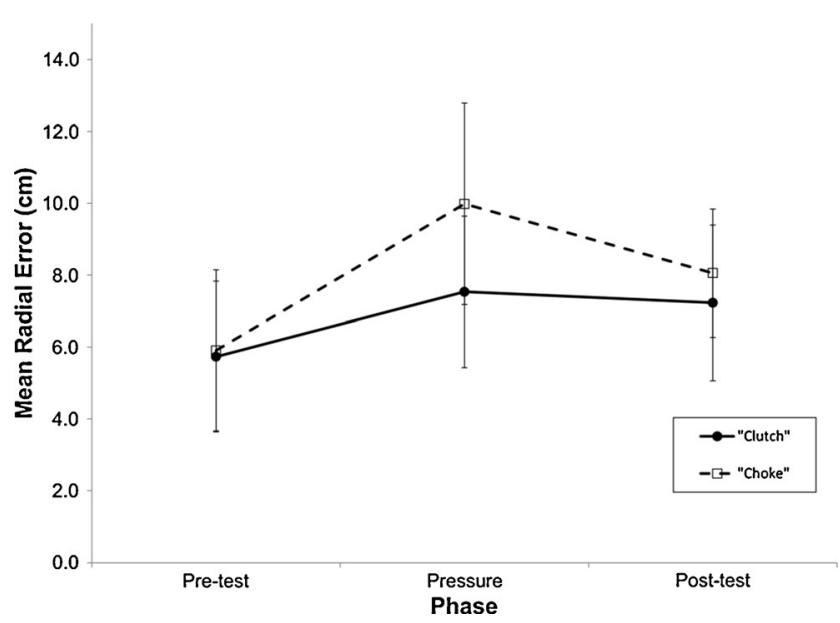

Fig. 2 Mean radial error (MRE) for the different phases of the experiment. Error bars show $\pm 1 \mathrm{SE}$

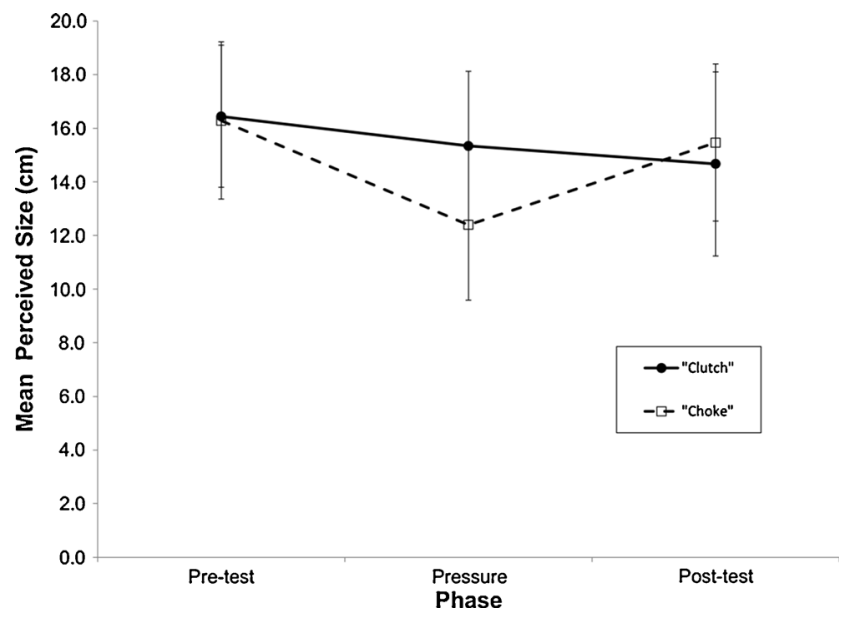

Fig. 3 Mean perceived size (MPS) for the different phases of the experiment. Error bars show $\pm 1 \mathrm{SE}$

\section{Secondary task performance}

Finally, Fig. $4 \mathrm{a}$ and $\mathrm{b}$ show respectively $\%$ Hole and $\% \mathrm{Club}$ for the two groups across the different phases of the experiment. The $2 \times 3$ ANOVA performed on the $\%$ Hole data revealed a
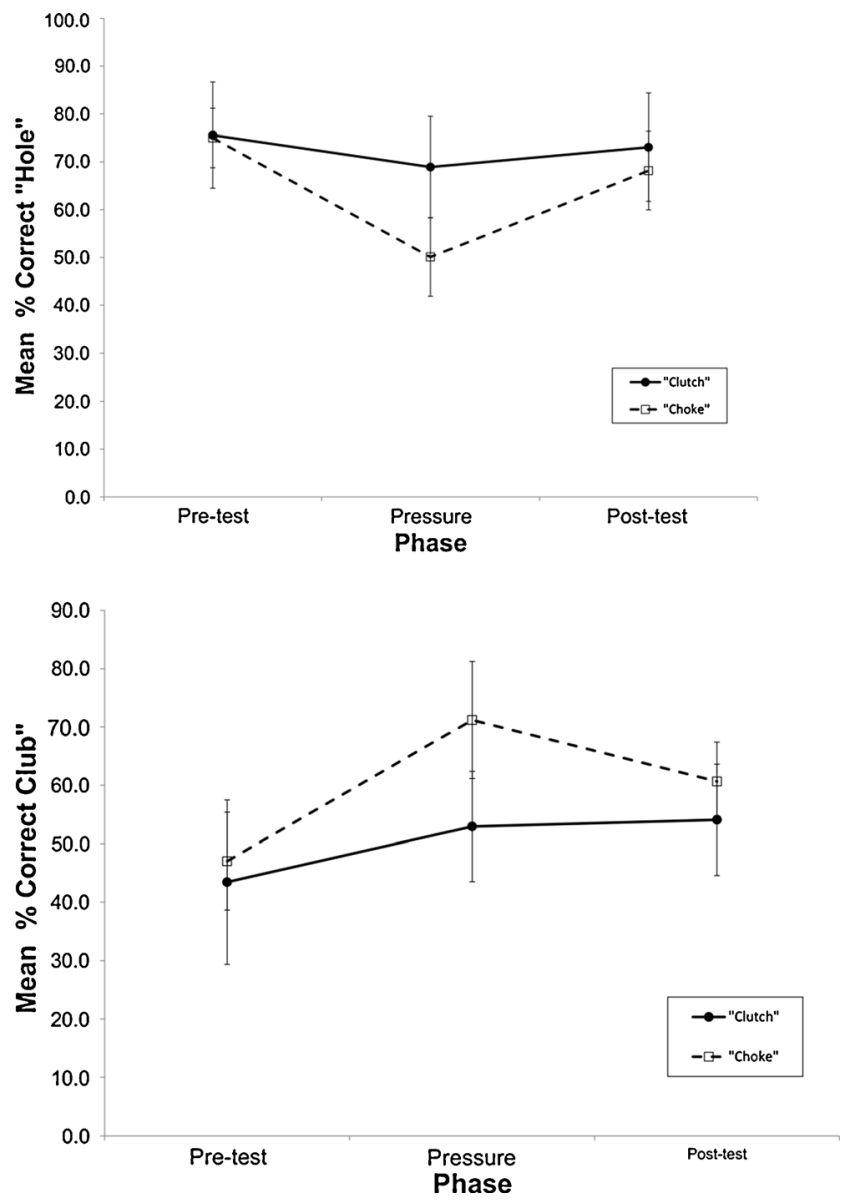

Fig. 4 Mean percentage correct in the Hole (top) and Club (bottom) secondary tasks across the different phases of the experiment. Error bars show $\pm 1 \mathrm{SE}$. See text for details 
significant main effects of phase $[\mathrm{F}(2,46)=16.3, p<0.001$, $\left.\eta_{\mathrm{p}}^{2}=0.42\right]$ and group $\left[\mathrm{F}(1,23)=14.7, p=0.001, \eta_{\mathrm{p}}^{2}=0.36\right]$ and a significant group $\mathrm{x}$ phase interaction $[\mathrm{F}(2,46)=5.7$, $\left.p=0.006, \eta_{\mathrm{p}}^{2}=0.20\right]$. Post-hoc $t$ tests revealed that \%Hole was significantly lower in pressure phase for Choke group: $\mathrm{t}(23)=-4.9, p=0.00$. All other comparisons were not significant. The $2 \times 3$ ANOVA performed on the $\%$ Club data revealed a significant main effects of phase $[\mathrm{F}(2,46)=23.0$, $\left.p<0.001, \eta_{\mathrm{p}}^{2}=0.50\right]$ and group $[\mathrm{F}(1,23)=11.3, p=0.003$, $\left.\eta_{\mathrm{p}}^{2}=0.33\right]$ and a significant group $\mathrm{x}$ phase interaction $\left[\mathrm{F}(2,46)=4.6, p=0.01, \eta_{\mathrm{p}}^{2}=0.17\right]$. Post-hoc $t$ tests revealed $\%$ Club significantly higher in pressure phase for Choke group: $\mathrm{t}(23)=-4.66, p<0.001$. There also was a marginally significant difference in post-pressure phase: $\mathrm{t}(23)=-2.0, p=0.05$.

\section{Discussion}

Despite the abundant evidence demonstrating that the action capability of a performer can influence the perceived size of the object(s) that they are acting on, to date there is no clear agreement as to which specific mechanism underlies these effects (see Firestone 2013 vs. Proffitt 2013). One key question that has remained unanswered is what the role is of attention in these effects? Do objects look bigger to performers of higher skill level (or performers that have recently been successful), because they are better able to maintain their focus of attention on the target object and this attention serves to accentuate perception? As discussed above, although previous studies are consistent with the proposal that action-specific effects are mediated by attention (Cañal-Bruland and van der Kamp 2009; Cañal-Bruland et al. 2011; Gray 2013; Gray et al. 2014), a direct link has yet to be established. The primary goal of the present study was to further investigate this issue by taking advantage of an effect that has been reported in several previous studies on skilled motor performance (reviewed in Beilock and Gray 2007), namely choking under pressure. That is, the introduction of performance pressure often leads to an inward shift in a performer's focus of attention. By assessing perceived hole size, performance outcome, and attentional focus in a golf putting task, we sought to establish a more direct link between these variables. A secondary goal of the present study was to investigate the relationship between movement kinematics and perceived target size. The vast majority of studies in this area have only looked at performance outcomes (Witt and Proffitt 2005; Cañal-Bruland and van der Kamp 2009; Lee et al. 2012; Witt et al. 2008). It hence remained to be determined whether changes to the way in which a performer is executing an action are associated with changes in perceived size of the goal object.

Consistent with previous research, the addition of pressure resulted in a significant change in the movement kinematics for some (but not all) golfers in the present study (Gray et al. 2013). Specifically, for 11 of 25 golfers, there was a significant decrease in TTPS (and increase in VI). A shorter TTPS typically occurs when a golfer has a very symmetrical putting stroke such that the maximum velocity occurs exactly at the point of ball-club contact: a technique commonly used by novice golfers (Delay et al. 1997). Expert golfers, on the other hand, typically use an asymmetrical putting stroke for which the club-head is still accelerating at the point of contact with the ball, and thus, TTPS (measured relative to start of the stroke) occurs a bit later (Delay et al. 1997). The advantage of using an asymmetric putting stroke with a longer TTPS is that the ball maintains contact with the club-head for a short period time after the moment of initial impact which results in the ball rolling more smoothly across the green (Pelz 2000). Therefore, the decrease in TTPS for the golfers in the Choke group in the present study is consistent with a regression to an earlier stage of skill acquisition as proposed by reinvestment theories of choking under pressure (Masters 1992). This idea is further supported by the finding (Fig. 2) that MRE was significantly higher in the pressure phase for the Choke group.

Also consistent with previous research, this change in putting kinematics in the pressure phase was associated with an inward shift of attentional focus, i.e., towards skill execution and away from the external environment (Baumeister 1984; Beilock and Gray 2012). As shown in Fig. 4, golfers in the Choke group had a significant decrease in accuracy for the external, secondary task that involved making a judgment relative to the hole location and a significant increase in the accuracy of the internal, skill-focused secondary task that involved making a judgment about club movement. Together, these findings provide further support for explicit monitoring (and related) theories of choking under pressure in which it is proposed that pressure serves to cause inward shift in attention towards skill execution resulting a perceptual-motor control strategy typical of an earlier stage of skill acquisition (Baumeister 1984).

Turning to the dependent variable that was the main interest of the present study, as shown in Fig. 3, the addition of pressure led to a significant decrease in the perceived size of the hole for the Choke group, whereas there was no significant change for the Clutch group. These findings extend the previous work of Cañal-Bruland et al. (2011) who demonstrated that the introduction of an attention-demanding secondary task to golf putting results in a nonsignificant relationship between putting performance and perceived hole size (i.e., no action-specific effect on perception). These results provide evidence consistent with the attentional accentuation hypothesis for action-specific effects (Cañal-Bruland and van der Kamp 2009). For similar links between attention, motor performance, and perceived size see Wood et al. (2013).

There were some important design choices in the present study that could have influenced the pattern of results and 
should be examined further in future research. First, participants were allowed to see the final position of the ball after each putt. As proposed by Proffitt and Linkenauger (2013), this knowledge of results could have provided a scaling metric for the perceived hole size judgments (i.e., the size estimate was related to the variability of final ball locations). However, although there is some indirect evidence in support of this hypothesis (Cañal-Bruland et al. 2012), in a more recent study we have shown that when performance variability was directly manipulated perceived target size remained stable independent of performance outcome variability (Foerster et al. 2015). Regardless, it will be important for future studies to examine whether the effects observed in the present study occur when performers are not given knowledge of results as has been done in previous studies (Wood et al. 2013).

Another design-related issue concerns the timing of the secondary task. In the present study, the secondary task stimuli used to assess attentional focus were presented during the putting stroke to allow for direct comparison with our previous research (Beilock and Gray 2012). Therefore, performance on these tasks could not be used to assess directly the attentional focus before the initiation of the putt. It is possible that the judged size of the target was determined by the attentional focus of the performer before movement initiation, which could have potentially been different than the focus during the stroke. However, we argue that this was likely not the case. First, previous research on golfer's eye movements (which can be used as an in direct measure of attentional focus) has shown that fixation patterns are similar before, during, and after the putting stroke (Vickers 1992). Second, we have shown previously by using an analogous secondary task in a baseball batting experiment that attentional shifts that are induced during the movement can spill over into the behavior that occurs before and after movement (Gray 2006).

In conclusion, the present study helps to advance understanding of the mechanisms underlying action-specific effects on perception. We provide direct evidence linking the perceived size of a target with extent to which an actor focuses their attention on that object-a finding that supports the attentional accentuation hypothesis. Finally, we show how the relationship between attentional focus and perceived size is accompanied by changes in putting kinematics.

\section{References}

Balcetis, E., \& Dunning, D. (2010). Wishful seeing: Desired objects are seen as closer. Psychological Science, 21, 147-152.

Baumeister, R. F. (1984). Choking under pressure - Self-consciousness and paradoxical effects of incentives on skillful performance. Journal of Personality and Social Psychology, 46, 610-620. doi: 10.1037/0022-3514.46.3.610
Beilock, S. L., \& Gray, R. (2007). Why do athletes "choke" under pressure? In G. Tenenbaum \& B. Eklund (Eds.), Handbook of sport psychology (rth ed., pp. 425-444). New Jersey: Wiley.

Beilock, S. L., \& Gray, R. (2012). From attentional control to attentional spillover: A skill-level investigation of attention, movement, and performance outcome relations. Human Movement Science, 31, $1473-1499$.

Bruner, J. S. (1957). On perceptual readiness. Psychological Review, 64, $123-152$.

Cañal-Bruland, R., \& van der Kamp, J. (2009). Action goals influence action-specific perception. Psychonomic Bulletin \& Review, 16, $1100-1105$.

Cañal-Bruland, R., Pijpers, J. R., \& Oudejans, R. R. D. (2010). The influence of anxiety on action-specific perception. Anxiety, Stress \& Coping, 23, 353-361.

Cañal-Bruland, R., Zhu, F. F., van der Kamp, J., \& Masters, R. S. W. (2011). Target-directed visual attention is a prerequisite for actionspecific perception. Acta Psychologica, 136, 285-289.

Cañal-Bruland, R., Pijpers, J. R., \& Oudejans, R. R. D. (2012). Close, and a cigar! Why size perception relates to performance. Perception, 41, 354-356.

Cooke, A., Kavussanu, M., McIntyre, D., \& Ring, C. (2010). Psychological, muscular and kinematic factors mediate performance under pressure. Psychophysiology, 47, 1109-1118.

Cooper, A. D., Sterling, C. P., Bacon, M. P., \& Bridgeman, B. (2012). Does action effect perception or memory? Vision Research, 62, 235-240.

Delay, D., Nougier, V., Orliaguet, J. P., \& Coello, Y. (1997). Movement control in golf putting. Human Movement Science, 16, 597-619.

Durgin, F. H., Baird, J. A., Greenburg, M., Russell, R., Shaughnessy, K., \& Waymouth, S. (2009). Who is being deceived? The experimental demands of wearing a backpack". Psychonomic Bulletin \& Review, 16, 964-969.

Firestone, C. (2013). How "paternalistic" is spatial perception? Why wearing a heavy back doesn't - and couldn't - make hills look steeper. Perspectives on Psychological Science, 8, 455-473.

Foerster, A., Gray, R., \& Cañal-Bruland, R. (2015). Size estimates remain stable in the face of differences in performance outcome variability in an aiming task. Consciousness and Cognition, 33, 47-52.

Gibson, J. J. (1950). The perception of the visual world. Boston, MA: Houghton Mifflin.

Gray, R. (2004). Attending to the execution of a complex sensorimotor skill: Expertise differences, choking and slumps. Journal of Experimental Psychology: Applied, 10, 42-54.

Gray, R. (2006). Expertise differences in attentional control between and within baseball batters. Proceedings of the Human Factors and Ergonomics Society 50th Annual Meeting, San Francisco, CA. pp. $1652-1655$.

Gray, R. (2013). Being selective at the plate: Processing dependence between perceptual variables relates to hitting goals and performance. Journal of Experimental Psychology: Human Perception and Performance, 39, 1124-1142.

Gray, R., \& Allsop, J. (2013). Interactions between performance pressure, performance streaks and attentional focus. Journal of Sport \& Exercise Psychology, 35, 368-386.

Gray, R., Allsop, J., \& Williams, S. (2013). Changes in putting kinematics associated with choking and excelling under pressure. International Journal of Sport Psychology (Performance under Pressure Special Issue), 44, 387-407.

Gray, R., Antonio Navia, J., \& Allsop, J. (2014). Action specific effects in aviation: What determines judged runway size? Perception, 43, $145-154$.

Lee, Y., Lee, S., Carello, C., \& Turvey, M. T. (2012). An archer's perceived form scales the "hitableness" of archery targets. Journal of Experimental Psychology: Human Perception and Performance, $38,1125-1131$. 
Lohse, K. R., Sherwood, D. E., \& Healy, A. F. (2010). How changing the focus of attention affects performance, kinematics and electromyography in dart throwing. Human Movement Science, 29, 542-555.

Masters, R. S. W. (1992). Knowledge, knerves and know-how-The role of explicit versus implicit knowledge in the breakdown of a complex motor skill under pressure. British Journal of Psychology, 83, 343-358.

Otten, M. (2009). Choking vs. clutch performance: A study of sport performance under pressure. Journal of Sport \& Exercise Psychology, $31,583-601$.

Pelz, D. T. (2000). Dave Pelz's putting bible: The complete guide to mastering the green. London: Aurum Press Ltd.

Proffitt, D. R. (2006). Embodied perception and the economy of action. Perspectives on Psychological Science, 1, 110-122.

Proffitt, D. R. (2013). An embodied approach to perception: By what units are visual perceptions scaled? Perspectives on Psychological Science, 8, 474-483.

Proffitt, D., \& Linkenauger, S. (2013). Perception viewed as a phenotypic expression. In W. Prinz, M. Beisert, \& A. Herwig (Eds.), Action science: foundations of an emerging discipline (pp. 171-198). Cambridge: MIT Press.
Thomas, O., Hanton, S., \& Jones, G. (2002). An alternative approach to short-form self-report assessment of competitive anxiety. International Journal of Sport Psychology, 33, 325-336.

Vickers, J. N. (1992). Gaze control in putting. Perception, 21, 117-132.

Wesp, R., Cichello, P., Gracia, E. B., \& Davis, K. (2004). Observing and engaging in purposeful actions with objects influences estimates of their size. Perception \& Psychophysics, 66, 1261-1267.

Witt, J. K., \& Dorsch, T. E. (2009). Kicking to bigger uprights: Field goal performance influences perceived size. Perception, $38,1328-1340$.

Witt, J. K., \& Proffitt, D. R. (2005). See the ball, hit the ball - Apparent ball size is correlated with batting average. Psychological Science, 16, 937-938.

Witt, J. K., Linkenauger, S. A., Bakdash, J. Z., \& Proffitt, D. R. (2008). Putting to a bigger hole: Golf performance relates to perceived size. Psychonomic Bulletin \& Review, 15, 581-585.

Wood, G., Vine, S. J., \& Wilson, M. R. (2013). The impact of visual illusions on perception, action planning, and motor performance. Attention, Perception, \& Psychophysics, 75, 830-834. 